\title{
Variability in the point to which single direct field irradiation is prescribed for spinal bone metastases: a survey of practice patterns in Japan
}

\author{
Naoki NAKAMURA ${ }^{1, *}$, Naoto SHIKAMA ${ }^{2}$, Hitoshi WADA ${ }^{3}$, Hideyuki HARADA ${ }^{4}$, \\ Miwako NOZAKI ${ }^{5}$, Hisayasu NAGAKURA ${ }^{6}$, Masao TAGO ${ }^{7}$, \\ Masahiko $\mathrm{OGUCHI}^{8}$ and Nobue $\mathrm{UCHIDA}^{9}$
}

\begin{abstract}
${ }^{1}$ Department of Radiation Oncology, St Luke's International Hospital, 9-1, Akashicho, Chuouku, Tokyo, 104-8560, Japan ${ }^{2}$ Department of Radiation Oncology, Saitama Medical University International Medical Center, 1397-1, Hidaka, 350-1298, Japan

${ }^{3}$ Department of Radiation Oncology, Miyagi Cancer Center, 47-1, Nodayama, Medeshimashiote, Natori, 981-1293, Japan

${ }^{4}$ Division of Radiation Oncology, Shizuoka Cancer Center, 1007 Shimonagakubo, Nagaizumi-cho, Sunto-gun, Mishima, 411-8777, Japan

${ }^{5}$ Department of Radiology, Dokkyo Medical University Koshigaya Hospital, 2-1-50, Minamikoshigaya, Koshigaya, 343-8555, Japan

${ }^{6}$ Department of Radiology, KKR Sapporo Medical Center, 6-3-40, Ichijo, Hiragishi, Tohiraku, Sapporo, 062-0931, Japan

${ }^{7}$ Department of Radiology, Teikyo University School of Medicine University Hospital, Mizonokuchi, 3-8-3, Mizonokuchi, Takatsuku, Kawasaki, 213-8507, Japan

${ }^{8}$ Department of Radiation Oncology, Cancer Institute Hospital of the Japanese Foundation for Cancer Research, 3-8-31, Ariake, Kotoku, Tokyo, 135-8550, Japan

${ }^{9}$ Department of Radiology, Tottori Chuoh Hospital, 730, Gotsu, Tottori, 680-0901, Japan

*Corresponding author. Department of Radiation Oncology, St Luke's International Hospital, 9-1, Akashicho, Chuouku, Tokyo, 104-8560, Japan. Tel: +81-3-3541-5151; Fax: +81-3-3544-0649; Email: naokinak@luke.or.jp
\end{abstract}

(Received 27 February 2013; revised 21 March 2013; accepted 22 March 2013)

\begin{abstract}
Direct single fields are commonly used in radiotherapy for spinal bone metastases, and it is crucial to define the point for which the dose is prescribed. According to the guidelines from the International Bone Metastases Consensus Working Party (IBMCWP) updated in 2010, different opinions exist on whether this therapy should be prescribed to the mid-vertebral or anterior vertebral body. To our knowledge, no previous studies have surveyed practice patterns regarding this discrepancy. Therefore, we performed an Internet-based survey of members of the Japanese Radiation Oncology Study Group (JROSG) to investigate the current practice patterns in Japan. The respondents mentioned the point to which they prescribed radiotherapy for a single direct field. A total of 52 radiation oncologists from 50 institutions (36\% of JROSG institutions) responded. Respondent prescription for radiotherapy varied widely. Only $21 \%$ and $6 \%$ of respondents prescribed irradiation to the mid-vertebral body and anterior vertebral body, respectively. A larger proportion of respondents (27\%) prescribed irradiation to the spinal cord (center of the spinal canal). Still another group of respondents $(27 \%)$ stated that they never use a single direct field. In conclusion, the point to which irradiation dosages are prescribed varies widely for a single direct field in cases of spinal bone metastases. This variation may lead some radiation oncologists to misunderstand the tolerance dosage of the spinal cord, especially in cases of reirradiation. Thus, careful consideration is required before any prescriptions are made.
\end{abstract}

Keywords: radiotherapy; bone metastases; spine; patterns of care study; prescription point 


\section{INTRODUCTION}

Direct single fields are commonly used in radiotherapy for thoracic and lumbar spinal bone metastases [1]. It is crucial to define the point to which the dose is prescribed. Variations of the point cause changes in doses for the tumor and organs at risk, particularly for the spinal cord, which may lead to variations in the efficacy and safety of radiotherapy for spinal bone metastases [2]. According to the guidelines from the International Bone Metastases Consensus Working Party (IBMCWP) updated in 2010, different opinions exist on whether this therapy should be prescribed to the midvertebral or anterior vertebral body [3]. However, to our knowledge, no previous studies have surveyed practice patterns regarding this discrepancy. Therefore, we investigated the current practice patterns in Japan.

\section{MATERIALS AND METHODS}

Members of the Japanese Radiation Oncology Study Group (JROSG), all of whom were radiation oncologists, completed an Internet-based survey from December 2010 to January 2011. Respondents were asked to define the point to which the dose is prescribed for a single direct field in a case of spinal bone metastases, in addition to the radiotherapy dose fractionation they would recommend for hypothetical cases describing patients with painful bone metastases [4]. As a reference for the variability of irradiated dose, we calculated monitor unit ratios for each point at which respondents prescribed radiotherapy compared to cases prescribed for the

Table 1. Points where respondents prescribed radiotherapy for a single direct field for spinal bone metastases $(n=52)$

\begin{tabular}{llc}
\hline Points & $\begin{array}{c}\text { Monitor Unit } \\
\text { ratio }^{\mathbf{a}}\end{array}$ & $\begin{array}{c}\text { Number } \\
(\%)\end{array}$ \\
\hline Anterior vertebral body & 1.10 & $3(6 \%)$ \\
Mid-vertebral body & 1 (referent) & $11(21 \%)$ \\
$\begin{array}{l}\text { Posterior vertebral body } \\
\text { Spinal cord (center of the spinal } \\
\quad \text { canal) }\end{array}$ & 0.92 & $2(4 \%)$ \\
$\begin{array}{l}\text { 3-cm depth } \\
\text { 6-cm depth }\end{array}$ & 0.81 & $14(27 \%)$ \\
Maximum dose point & 0.94 & $4(8 \%)$ \\
$\begin{array}{l}\text { Center of the gross tumor volume } \\
\text { Adjust the point, considering dose }\end{array}$ & 0.94 & $1(2 \%)$ \\
$\quad$ distribution & & $1(2 \%)$ \\
Never use a single direct field & & $14(27 \%)$ \\
\hline $\begin{array}{l}\text { a Forty simulation CT scans } \\
\text { thoraco-lumbar spinal bone metastases were used for the } \\
\text { calculation. }\end{array}$ &
\end{tabular}

mid-vertebral body, using 40 simulation CT scans involving patients previously treated for thoraco-lumbar spinal bone metastases.

\section{RESULTS}

A total of 52 radiation oncologists from 50 institutions (36\% of JROSG institutions) responded. Of those, 32 respondents $(62 \%)$ work at university hospitals or cancer centers, 15 (29\%) at public hospitals, and $5(10 \%)$ at private hospitals.

Respondent prescription for radiotherapy for a single direct field for spinal bone metastases varied widely (Table 1). Monitor unit ratios ranged from 0.76-1.10 relative to that prescribed for the mid-vertebral body. Only $21 \%$ and $6 \%$ of respondents prescribed irradiation to the mid-vertebral body and anterior vertebral body, respectively. A larger proportion of respondents $(27 \%)$ prescribed irradiation to the spinal cord (center of the spinal canal). Still another group of respondents (27\%) stated that they never use a single direct field.

\section{DISCUSSION}

Large variations were found amongst respondents prescribing radiotherapy for a single direct field. These variations cause changes in doses for the tumor and organs at risk, which may lead to variations in the efficacy and safety [2]. Furthermore, the variations may make it difficult to clarify the tolerance dose of the spinal cord in cases of re-irradiation. While recurrent pain in spinal bones can be successfully alleviated with external beam radiotherapy re-treatment, optimal dosage and fractionation are still under investigation [5]. Nieder et al. collected data from 40 individual patients who received re-irradiation of the spinal cord, published in eight different reports, and mentioned that the risk of radiationinduced myelopathy appears to be small after cumulative doses of $\leq 135.5 \mathrm{~Gy}_{2}$ when the interval is not less than 6 months and the dose of each course is $\leq 98 \mathrm{~Gy}_{2}$ [6]. However, the cumulative dose was calculated from prescribed doses without the absolute correctness of the doses to the spinal cord.

According to the guideline from the IBMCWP, updated in 2010 , opinion was split between prescribing to the midvertebral body or anterior vertebral body [3]. However, only 21 and $6 \%$ of respondents prescribed irradiation to the midvertebral body and anterior vertebral body, respectively. Japanese radiation oncologists may be concerned that prescribing irradiation to the mid-vertebral body or anterior vertebral body may cause too high a dose to the spinal cord.

A larger proportion of respondents prescribed irradiation to the spinal canal (center of the spinal canal). A recent large multi-institutional randomized controlled trial (RCT) prescribed irradiation to the posterior edge of the vertebral body [7] (Table 2). The monitor units we calculated were similar 
The point to which single direct field irradiation is prescribed

between the prescription to the posterior vertebral body and to the spinal cord. Furthermore, some RCTs prescribed to a 5-cm depth, which was probably close to the spinal canal. Therefore, it seems to be reasonable to prescribe irradiation to the spinal canal if we do not consider the guideline of the IBMCWP [1].

Although the efficacy and safety of radiotherapy using a single direct field for spinal bone metastases have been proven through numerous clinical trials for both single- and multi-fraction radiotherapy [7-15], a large number of respondents stated that they never use a single direct field for spinal bone metastases. Those who never use a single direct field may prefer to use parallel opposing fields or highly conformal radiotherapy. Parallel opposed fields give a more homogenous dose distribution, which can avoid overdosing the spinal cord or underdosing the tumor. Stereotactic body radiotherapy (SBRT) is a technology that delivers high doses to spinal metastases with a steep dose gradient, which might allow superior sparing of the adjacent organs at risk, including the spinal cord. However, the efficacy and safety of SBRT have not been fully evaluated yet, and the ASTRO evidence-based guideline published in 2011 strongly suggests that SBRT should only be used within clinical trials [16].

Our study has certain limitations. Due to the relatively low response rate $(36 \%)$ and small absolute sample size $(n=52)$, our results might not accurately represent the practice of radiation oncologists in Japan. Furthermore, those willing to participate might have been more knowledgeable than those unwilling to participate.

In conclusion, the point to which irradiation dosages are prescribed varies widely for a single direct field in cases of spinal bone metastases. This variation may lead some radiation oncologists to misunderstand the tolerance dosage of the spinal cord, especially in cases of re-irradiation. Thus, careful consideration is required before any prescriptions are made.

\section{ACKNOWLEDGEMENTS}

We thank all the radiation oncologists who participated in this study. The results of this study have been presented in part at the 54th Annual Meeting of the American Society for Radiation Oncology (ASTRO) in Boston, MA, 2012. All authors are part of the JROSG Working Subgroup of Palliative Radiotherapy.

\section{REFERENCES}

1. Chow E, Wu JS, Hoskin P et al. International consensus on palliative radiotherapy endpoints for future clinical trials in bone metastases. Radiother Oncol 2002;64:275-80.

2. Hoskin PJ, Yarnold JR, Roos DR et al. Radiotherapy for bone metastases. Clin Oncol (R Coll Radiol) 2001;13:88-90. 
3. Chow E, Hoskin P, Mitera G et al. Update of the International Consensus on Palliative Radiotherapy Endpoints for Future Clinical Trials in Bone Metastases. Int J Radiat Oncol Biol Phys 2012;82:1730-7.

4. Nakamura N, Shikama N, Wada $\mathrm{H}$ et al. Patterns of practice in palliative radiotherapy for painful bone metastases: a survey in Japan. Int J Radiat Oncol Biol Phys 2012;83:e117-20.

5. Chow E, Hoskin PJ, Wu J et al. A phase III international randomised trial comparing single with multiple fractions for re-irradiation of painful bone metastases: National Cancer Institute of Canada Clinical Trials Group (NCIC CTG) SC 20. Clin Oncol ( $R$ Coll Radiol) 2006;18:125-8.

6. Nieder C, Grosu AL, Andratschke NH et al. Proposal of human spinal cord reirradiation dose based on collection of data from 40 patients. Int J Radiat Oncol Biol Phys 2005;61:851-5.

7. Kaasa S, Brenne E, Lund JA et al. Prospective randomised multicenter trial on single fraction radiotherapy $(8 \mathrm{~Gy} \times 1)$ versus multiple fractions $(3 \mathrm{~Gy} \times 10)$ in the treatment of painful bone metastases. Radiother Oncol 2006;79:278-84.

8. Price P, Hoskin PJ, Easton D et al. Prospective randomised trial of single and multifraction radiotherapy schedules in the treatment of painful bony metastases. Radiother Oncol 1986;6:247-55.

9. Gaze MN, Kelly CG, Kerr GR et al. Pain relief and quality of life following radiotherapy for bone metastases: a randomized trial of two fractionation schedules. Radiother Oncol 1997;45:109-16.
10. Nielsen OS, Bentzen SM, Sandberg E et al. Randomized trial of single dose versus fractionated palliative radiotherapy of bone metastases. Radiother Oncol 1998;47:233-40.

11. Bone Pain Trial Working Party. 8 Gy single fraction radiotherapy for the treatment of metastatic skeletal pain: randomised comparison with a multifraction schedule over 12 months of patient follow-up. Radiother Oncol 1999; 52:111-21.

12. Steenland E, Leer JW, van Houwelingen $\mathrm{H}$ et al. The effect of a single fraction compared to multiple fractions on painful bone metastases: a global analysis of the Dutch Bone Metastasis Study. Radiother Oncol 1999;52:101-9.

13. Hartsell WF, Scott CB, Bruner DW et al. Randomized trial of short- versus long-course radiotherapy for palliation of painful bone metastases. J Natl Cancer Inst 2005;97: 798-804.

14. Roos DE, Turner SL, O'Brien PC et al. Randomized trial of 8 Gy in 1 versus $20 \mathrm{~Gy}$ in 5 fractions of radiotherapy for neuropathic pain due to bone metastases (Trans-Tasman Radiation Oncology Group, TROG 96.05). Radiother Oncol 2005;75:54-63.

15. Chow E, Zeng L, Salvo N et al. Update on the systemic review of palliative radiotherapy trials for bone metastases. Clin Oncol ( $R$ Coll Radiol) 2012;24:112-24.

16. Lutz S, Berk L, Chang E et al. Palliative radiotherapy for bone metastases: an ASTRO evidence-based guideline. Int J Radiat Oncol Biol Phys 2011;79:965-76. 